\title{
Altered functional connectivity in adolescent anorexia nervosa is related to age and cortical thickness
}

\author{
Anna D. Myrvang ${ }^{1 *}$, Torgil R. Vangberg ${ }^{2,3}$, Clas Linnman $^{4}$, Kristin Stedal ${ }^{5}, \varnothing y v i n d ~ R \varnothing^{5,6}$, Tor Endestad ${ }^{7,8}$,
} Jan H. Rosenvinge ${ }^{1}$ and Per M. Aslaksen ${ }^{1,9}$

\begin{abstract}
Introduction: Functional networks develop throughout adolescence when anorexia nervosa (AN) normally debuts. In AN, cerebral structural alterations are found in most brain regions and may be related to the observed functional brain changes. Few studies have investigated the functional networks of the brain in adolescent AN patients.. The aim of this explorative study was to investigate multiple functional networks in adolescent AN patients compared to healthy age-matched controls $(\mathrm{HC})$ and the relationship with age, eating disorder symptoms and structural alterations.
\end{abstract}

Methods: Included were 29 female inpatients with restrictive AN, and 27 HC. All participants were between the ages of 12 to 18 years. Independent component analysis (ICA) identified 21 functional networks that were analyzed with multivariate and univariate analyses of components and group affiliation (AN vs $\mathrm{HC}$ ). Age, age $\times$ group interaction and AN symptoms were included as covariates. Follow-up correlational analyses of selected components and structural measures (cortical thickness and subcortical volume) were carried out.

Results: Decreased functional connectivity (FC) in AN patients was found in one cortical network, involving mainly the precuneus, and identified as a default mode network (DMN). Cortical thickness in the precuneus was significantly correlated with functional connectivity in this network. Significant group differences were also found in two subcortical networks involving mainly the hippocampus and the amygdala respectively, and a significant interaction effect of age and group was found in both these networks. There were no significant associations between FC and the clinical measures used in the study.

Conclusion: The findings from the present study may imply that functional alterations are related to structural alterations in selected regions and that the restricted food intake in AN patients disrupt normal age-related development of functional networks involving the amygdala and hippocampus.

Keywords: Eating disorders, Anorexia nervosa, Adolescent, RS-fMRI

\footnotetext{
* Correspondence: anna.d.myrvang@uit.no

'Department of Psychology, Faculty of Health Sciences, UiT The Artic University of Norway, Huginbakken 32, N-9037 Tromsø, Norway

Full list of author information is available at the end of the article
}

(c) The Author(s). 2021 Open Access This article is licensed under a Creative Commons Attribution 4.0 International License, which permits use, sharing, adaptation, distribution and reproduction in any medium or format, as long as you give appropriate credit to the original author(s) and the source, provide a link to the Creative Commons licence, and indicate if changes were made. The images or other third party material in this article are included in the article's Creative Commons licence, unless indicated otherwise in a credit line to the material. If material is not included in the article's Creative Commons licence and your intended use is not permitted by statutory regulation or exceeds the permitted use, you will need to obtain permission directly from the copyright holder. To view a copy of this licence, visit http://creativecommons.org/licenses/by/4.0/. The Creative Commons Public Domain Dedication waiver (http://creativecommons.org/publicdomain/zero/1.0/) applies to the data made available in this article, unless otherwise stated in a credit line to the data. 


\section{Introduction}

Structural and functional changes in the brain have frequently been revealed in patients with anorexia nervosa (AN), a severe eating disorder characterized by abnormally low body weight and a body image disturbance. Cerebral structural alterations are found to mainly involve reduction in gray matter (GM) in numerous brain regions, and several studies find that most cortical areas are affected [1-3]. Functional magnetic resonance imaging (fMRI) studies typically utilize stimulus paradigms to uncover brain activity related to AN characteristic traits such as body image disturbance [4] and food and taste aversion [5]. These studies have revealed altered activity in several brain regions and functional networks, improving our understanding of the neurobiological correlate to this disorder.

In recent years, it has become increasingly common to investigate brain activity while subjects are at rest, not responding to any stimuli in the scanner - so called resting state fMRI (RS-fMRI). RS-fMRI can be used to identify resting-state networks (RSNs) - spatially separated areas of the brain where the BOLD-signal is temporally correlated [6]. Several RSNs that are consistent across trials and studies have been identified [7]. The networks are linked to known cognitive domains such as vision, somatosensation and motor function. A much studied network is the default mode network (DMN) [8]. The DMN is found to correlate negatively with taskdriven activity in fMRI studies [9].

In RS-fMRI studies conducted in AN patients, several different analytical approaches have been utilized. Many studies have used seed-based approaches, which are useful to investigate areas of interest. However, such approaches rely on a priori hypotheses and can thus fail to detect alterations in unselected brain regions. Some studies have investigated selected RSNs that may be linked to core symptoms of the eating disorder such as visuospatial [10] and executive control networks [11] and suggest that altered connectivity in these networks contribute to disturbance in body image perception and excessive cognitive control, respectively.

AN typically has its debut in adolescence [12], during a period in development where drastic changes occur in the organization of brain networks, both internally within networks and between different RSNs [13]. During adolescence intra-network connectivity appears to increase and inter-network connectivity decreases, suggesting that the networks become more established and that communication between networks becomes more efficient with increasing age [13]. Particularly RSNs involving areas such as the precuneus, the cingulate cortex and the insula were found to gain increasing intranetwork connectivity during adolescence. AN often delays normal developmental processes such as the onset of puberty and may also delay structural and functional brain development. To our knowledge, no studies have investigated the relationship between alterations in brain networks and development in adolescent AN patients. Adolescent AN patients are found to have a greater GM volume reduction compared to adults AN patients [2], and there may be considerable spatial overlap between functionally and structurally altered regions. A structure-function relationship is suggested, but not established in adult AN [14]; Scaife et al. (2017) reported that GM morphometrics explained functional connectivity alterations [15], and de la Cruz (2021) found reduced connectivity in regions where cortical thickness was reduced in AN patients [16]. Two other studies did not detect such a relationship [10, 17]. Seidel et al. (2019) reported a decreased structure-function relationship in AN relative to $\mathrm{HC}$ [17]

As the structural alterations in AN appear to be occurring across most of the cortex and several subcortical regions [1-3], it is possible that networks in several anatomical areas are affected. A common method for investigating whole-brain connectivity is independent component analysis (ICA). ICA is data-driven and does not require a-priori selection of regions to examine. To our knowledge, only one study has conducted whole brain ICA in adolescent AN patients, examining all the known RSNs detected [18]. The authors found that increased functional connectivity in a fronto-parietal network and DMN were associated with problems with interoceptive awareness.

The aim of this study was to investigate multiple networks detected in our dataset, covering large parts of the cortex and some subcortical regions that may be related to eating disorder symptoms, such as visuospatial-, executive control- and default mode-networks. Furthermore, we investigate the relationship between functional networks and age in adolescent AN-patients compared to healthy controls $(\mathrm{HC})$. As a structure-function link may exist, we also aimed to examine the relationship between functional networks and structural measures (cortical thickness and subcortical volume) in relevant anatomical regions.

\section{Methods}

\section{Study design and sample}

Acutely ill patients admitted to one of two clinics (Regional Center for Eating Disorders at the University Hospital of North Norway in Troms $\varnothing$, and Oslo University Hospital). In total, 29 female patients with AN (Age: $M=$ 15.9 $\mathrm{SD}=1.7)$ and 27 gender and healthy age-matched controls (Age: $\mathrm{M}=16.1, \mathrm{SD}=1.9$ ) between the ages of 12 to 18 years were recruited for the study ( 8 patients and 8 controls were tested and scanned at the Oslo clinic and the rest were included in Tromsø). The HC participants 
were recruited from local high schools. All participants were scanned in the afternoon. The inpatient AN group were scanned after dinner but before supper at the hospital. However, the exact time between meal to scanning were not recorded for any of the participants but the scanning did not interfere with the meal plan for any of the patients. In the healthy control group, the scanning was performed between 3 and $8 \mathrm{pm}$. During scanning, all participants were asked to stay awake and keep their eyes open and fixate their gaze on a cross on the in-scanner screen.

Inclusion criteria for AN patients were DSM-5 criteria for restrictive AN (no history of binge-purge episodes), diagnosis set by a clinical specialist in psychology or psychiatry. Age-adjusted, standardized body mass index values (BMI-SDS) were calculated using Norwegian normative data from the Bergen Growth Study [19]. Exclusion criteria for all participants were neurological disorders and organic brain injury, developmental disorder, history of bulimia nervosa, schizophrenia, psychotic episodes, and the use of antipsychotic medication. Additional exclusion criteria for $\mathrm{HC}$ were lifetime or current eating disorders, $\mathrm{BMI}<17.5$ or obesity (BMI $>$ 30 ). The sample is the same as described in two previously published articles $[3,20]$.

\section{Image acquisition}

MR scanning was performed with a $3 \mathrm{~T}$ Siemens Magnetom Skyra Syngo MR D13C in Tromsø and a Phillips Achieva $3 \mathrm{~T}$ scanner in Oslo, both equipped with 64 channel head coils. At both sites, high-resolution 3D T1wheighted images were acquired. In Tromsø, we used a magnetization-prepared rapid gradient-echo (MPRAGE) sequence with the following parameters: Orientation $=\mathrm{Sa}-$ gittal; No. of slices $=176$; Voxel size $=1 \times 1 \times 1$; Slice thickness $=1 \mathrm{~mm}$; repetition time $(\mathrm{TR})=2300 \mathrm{~ms}$; echo time $(\mathrm{TE})=2.98 \mathrm{~ms}$; field of view $(\mathrm{FOV})=256 \times 256$; Flip angle $=9^{\circ}$; and inversion time $(\mathrm{TI})=900 \mathrm{~ms}$. In Oslo, a 3D-TFE sequence was used with the following parameters: Orientation $=$ Sagittal; No of slices $=184$; Voxel size $=1 \times$ $1 \times 1$; Slice thickness $=1 \mathrm{~mm}$; TR $=2300 \mathrm{~ms}$; $\mathrm{TE}=2.98 \mathrm{~ms}$; FOV $=256 \times 256$; Flip angle $=8^{\circ}$; and TI $=900 \mathrm{~ms}$.

The following parameters were used for functional imaging at both sites: Voxel size: $3 \times 3 \times 3$, matrix size: $80 \times$ 80, TR: $2500 \mathrm{~ms}$., TE: $30 \mathrm{~ms}$., acquisition order: interleaved (43 slices), no. volumes: 288. Scan-time for fMRI sequence was $12.08 \mathrm{~min}$.

A group analysis of the potential confounding effect of scan site (Oslo $>$ Troms $\varnothing)$ was conducted using participants from the $\mathrm{HC}$ group.

\section{Preprocessing and image analyses}

The functional and structural images were preprocessed using FSL FEAT (FSL ver. 5.0.11, fsl.fmrib.ox.ac.uk). The functional images were corrected for scan-to-scan motion, coregistered to the high-resolution anatomical image, warped to the MNI152 template and spatially smoothed with an $8 \mathrm{~mm}$ FWHM Gaussian filter. No temporal filtering was applied. Next, motion-related independent components were removed with ICAAROMA [21, 22].

The software Group Independent Component Analyses Toolbox (GIFT) was used to extract functional networks (components) from the dataset and all further analyses [23]. ICA applies blind source separation to extract statistically independent components in the dataset. Group ICA was performed on the preprocessed images with the Infomax algorithm. Based on results from several large sample RSN studies [7, 24-26] a decision was made to set component numbers to 25 . The module ICASSO implemented in GIFT was set to run the Infomax algorithm 10 times, as is recommended [27]. ICAS SO graphs were inspected and evaluated by their component stability/cluster quality index (Iq $>.80)$, representing the difference between intra and extra-cluster similarity, and visual inspection of component maps. Two of the authors (PMA and ADM) rated the components. This process is further described in the Supplementary material 1 . One noise-related component (activation outside the cortex and in the ventricles) was identified by visualization and excluded from further analyses. Two components seemingly representing auditory networks were also excluded from further analyses as we did not hypothesize an impact of AN core symptoms in such networks. One cerebellar network received a low score from the two raters and was also excluded from further analyses. The excluded components are presented in Supplemental Fig. 2.

\section{Statistical analyses}

Group difference in sample characteristics were investigated with Mann-Whitney U-Tests using IBM SPSS 26. Shapiro-Wilk tests were used to test normality of the sample characteristics, cortical thickness, and cortical volumes. Furthermore, visual inspection of Q-Q- and Boxplots was performed. Significant deviations from a normal distribution were found for all sample characteristics variables except age.

Multivariate group analyses were conducted on timecourses spectra and spatial maps of the selected 21 components (Supplemental Fig. 1), including age and age "group interaction term as covariates. In subsequent analyses steps, BMI-SDS, the two EDE-Q scales "Restriction" and "Concerns about figure" [28] were included as covariates in separate models. The two subscales were selected because they did not correlate as highly with each other as the remaining subscales and thought to capture different presentations of AN. All analyses were performed with the MANCOVAN toolbox implemented 
in GIFT software [26]. MANCOVAN performs backward selection of predictors (factors and covariates) by testing whether each predictor in the model explains variability in the multivariate response using a multivariate analysis of covariance (MANCOVA), and for the reduced model of significant predictors proceeds to perform univariate tests corrected for multiple comparisons [26]. The multivariate results determine the significant covariates used in univariate analyses for timecourses spectra and spatial maps. False discovery rate (FDR) correction is implemented in MANCOVAN for multiple comparison corrections. Results retaining $p<0.05$ after FDR were considered statistically significant. Estimates of effect sizes are shown by weighted Beta values (group coding: $0=\mathrm{AN}, 1=\mathrm{HC}$ ) for each significant covariate. In the MANCOVAN toolbox, Beta values are averaged using weighted mean activated number of voxels in the groups. Following group analyses, we investigated the relationship between significant components and structural measures (cortical thickness and volume) extracted with FreeSurfer software [29], version 6.0 (FS 6.0) [30, 31]; . This procedure has been described previously in $[3,20]$. To perform correlations between the significant RSN components and structural measures, the maximum activation (peak) value in the selected RSN networks was extracted from MANCOVAN to SPSS and correlated (Pearson correlations) with the mean thickness data from FreeSurfer corresponding to the anatomical location of the maximum activation in the network. The mean value of thickness from both hemispheres were used. Bonferroni corrections were applied to correct for multiple testing in the correlational analyses of structure - function. Structural data were parcellated with the Desikan-Kiliany atlas [32], and regions overlapping spatially with significant RSN's were selected for analyses.

\section{Results}

\section{Sample characteristic}

Table 1 shows sample characteristics and tests of group means for AN and HC. AN patients had significantly lower BMI and higher scores on self-report measures of eating disorder and depressive symptoms. Table 2 shows additional characteristics of the AN group only.

\section{Multivariate results}

Multivariate analyses of spatial maps showed that there was a significant group effect $(p<.05)$ in five networks (Fig. 1), when including age and the interaction term age"group as covariates. Including BMI-SDS in this model did not alter results. A significant effect of age and a significant interaction effect of group and age was found in three of these networks (C6, C15 and C24).
Table 1 Sample characteristics

\begin{tabular}{lllll}
\hline & $\begin{array}{l}\text { AN } \\
\text { Mean (SD) }\end{array}$ & $\begin{array}{l}\text { HC } \\
\text { Mean (SD) }\end{array}$ & U-value & $\boldsymbol{p}$ \\
\hline $\mathrm{N}$ & 29 & 27 & & \\
Age & $15.9(1.7)$ & $16.1(1.9)$ & .33 & .37 \\
BMI & $16.3(1.7)$ & $22.0(3.1)$ & 50 & $<.001$ \\
BMI-SDS & $-2.4(1.3)$ & $0.4(0.9)$ & 49 & $<.001$ \\
Left hand dominant & 2 & 2 & & \\
BDI II & $24.1(12.6)$ & $4.3(5.2)$ & 1166 & $<.001$ \\
EDE-Q restriction $^{\text {a }}$ & $3.3(1.9)$ & $0.3(0.5)$ & 1129 & $<.001$ \\
EDE-Q eating $^{\mathrm{a}}$ & $2.5(1.6)$ & $0.2(0.5)$ & 1127.5 & $<.001$ \\
EDE-Q weight $^{\mathrm{a}}$ & $3.2(1.7)$ & $0.7(0.8)$ & 1117.5 & $<.001$ \\
EDE-Q figure $^{\mathrm{a}}$ & $4.1(1.7)$ & $0.8(0.9)$ & 1148.5 & $<.001$ \\
EDE-Q $^{\text {alobal }}{ }^{\mathrm{a}}$ & $3.3(1.5)$ & $0.5(0.5)$ & 1155 & $<.001$ \\
\hline
\end{tabular}

Note: Mann-Whitney U-Test. BMI Body mass index, BMI-SDS Standardized BMI values based on Norwegian norms for children, $B D I$ Becks Depression

Inventory II, EDE-Q Eating Disorder Examination Questionnaire. ${ }^{\text {a } A N ~ N ~}=27$

The multivariate model including EDE-Q restriction scale as a covariate showed similar results with significant effects of group, age and age"group interaction in the same networks and an additional significant effect of EDE-Q on a fifth network (C17). However, the EDE-Q variables were not retained for univariate analyses and are not reported further.

\section{Univariate results}

Univariate results of spatial maps showed significant group difference in $\mathrm{C} 4$ a default mode network. Figure $2 \mathrm{a}$ shows that the group difference $(B=-3.1)$ is most prominent in the central part of network C4 (peak voxels coordinates: X: $-12, \mathrm{Y}:-56, \mathrm{Z}: 56)$. Univariate results of group*age showed a significant interaction effect in network $\mathrm{C} 6(\mathrm{~B}=-3.1)$ and $\mathrm{C} 24(\mathrm{~B}=1.1)$, the two subcortical networks with peak activation in the amygdala (X: -26, Y: -6, Z: - 20) and hippocampal areas (X: -30, Y: $-30, \mathrm{Z}:-16)$ (Fig. 2b and c). Results for the left amygdala network (C6) indicates that there is a positive relationship with group*age, indicating greater intra-network

Table $\mathbf{2}$ Characteristics of the AN group

\begin{tabular}{ll}
\hline & $\begin{array}{l}\text { AN } \\
\text { Mean (SD) }\end{array}$ \\
\hline $\mathrm{N}$ & 29 \\
BMI admission & $15.0(1.4)$ \\
BMI-increase $^{\mathrm{a}}$ & $0.9(0.5)$ \\
${\text { Drugs }(S S R I / G H)^{\mathrm{b}}}$ & 4 \\
Weeks admitted $_{\text {Time since first GP contact (years) }}$ & $4.6(4.2)$ \\
\hline
\end{tabular}

Note: ${ }^{\mathrm{B}} \mathrm{BMI}$ increase between admission and scan date. ${ }^{\mathrm{b}} 5$ subjects on serotonin reuptake inhibitor (SSRI), 2 on growth hormones (GH). Time since first GP contact $=$ Consultation concering eating disorder symptoms. 


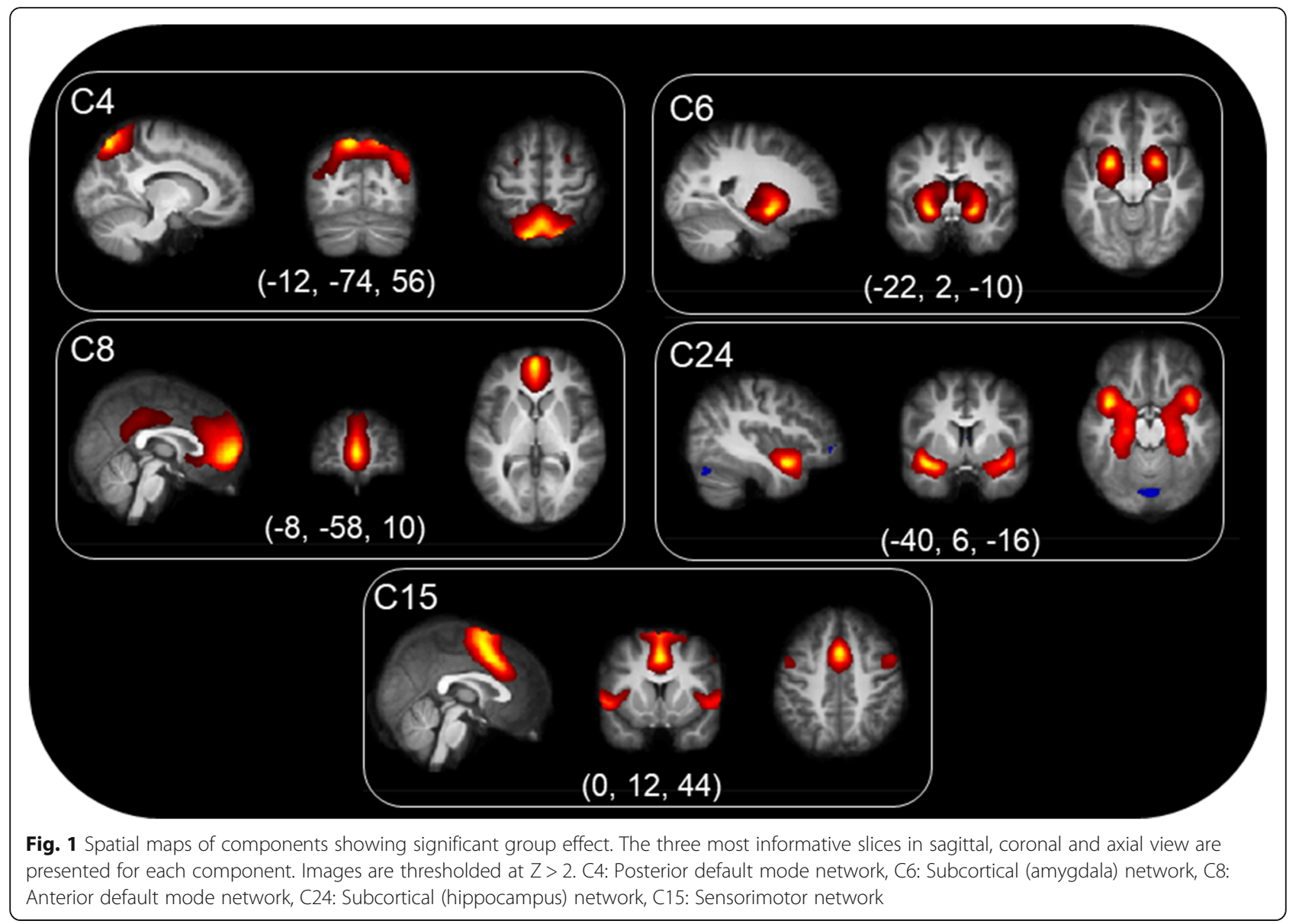

connectivity with increasing age in AN group (coded 1). Figure 2c shows that the significant interaction effect in C24 is negative, indicating decreasing intra-network connectivity in AN patients with increasing age compared to $\mathrm{HC}$.

\section{Correlation with structural measures}

Correlational analyses of structural measures were performed with the network that were significantly different between AN patients and controls, or had a significant interaction of group*age, namely C4, C6, and C24. The correlation analyses showed that precuneus thickness and component $\mathrm{C} 4$, the precuneus network, was significantly associated $(\mathrm{r}=.53, p<.001)$. The overlap between the precuneus area and the $\mathrm{C} 4$ component is shown in Fig. 3, whereas the correlation between $\mathrm{C} 4$ and precuneus thickness is shown in Fig. 4. Amygdala and hippocampal volumes were not significantly correlated with the components comprising these areas (component number $\mathrm{C} 6$ and $\mathrm{C} 24$ respectively).

\section{Control variables}

We performed a between-site (Oslo vs. Tromsø) analyses of $\mathrm{HC}$ participants to test for the effect of scanner site. To test for the effect of drug use, all analyses were re-performed controlling for/excluding the AN patients who were on prescribed drugs at the time of scanning $(N=5)$. We found no significant effect of scanner site or drug use.

\section{Discussion}

Compared with $\mathrm{HC}$, AN patients had decreased connectivity in a DMN network involving mainly the precuneus. Age affected two subcortical networks involving the hippocampus and amygdala differently for $\mathrm{AN}$ and $\mathrm{HC}$. In AN patients increasing age was associated with increasing connectivity within a network involving the amygdala and decreasing connectivity within a network involving the hippocampus. Precuneus thickness, found in our previously published study [3] to be reduced in AN compared to $\mathrm{HC}$, was significantly associated with connectivity in the DMN (precuneus) network.

The precuneus is a parietal region bordering to the visual cortex and is considered to be a functional core of the DMN [33]. In AN patients functional alterations are found repeatedly in this region, and have been linked to body image perception [4, 34-36] most often in terms of reduced activity or altered connectivity with other 


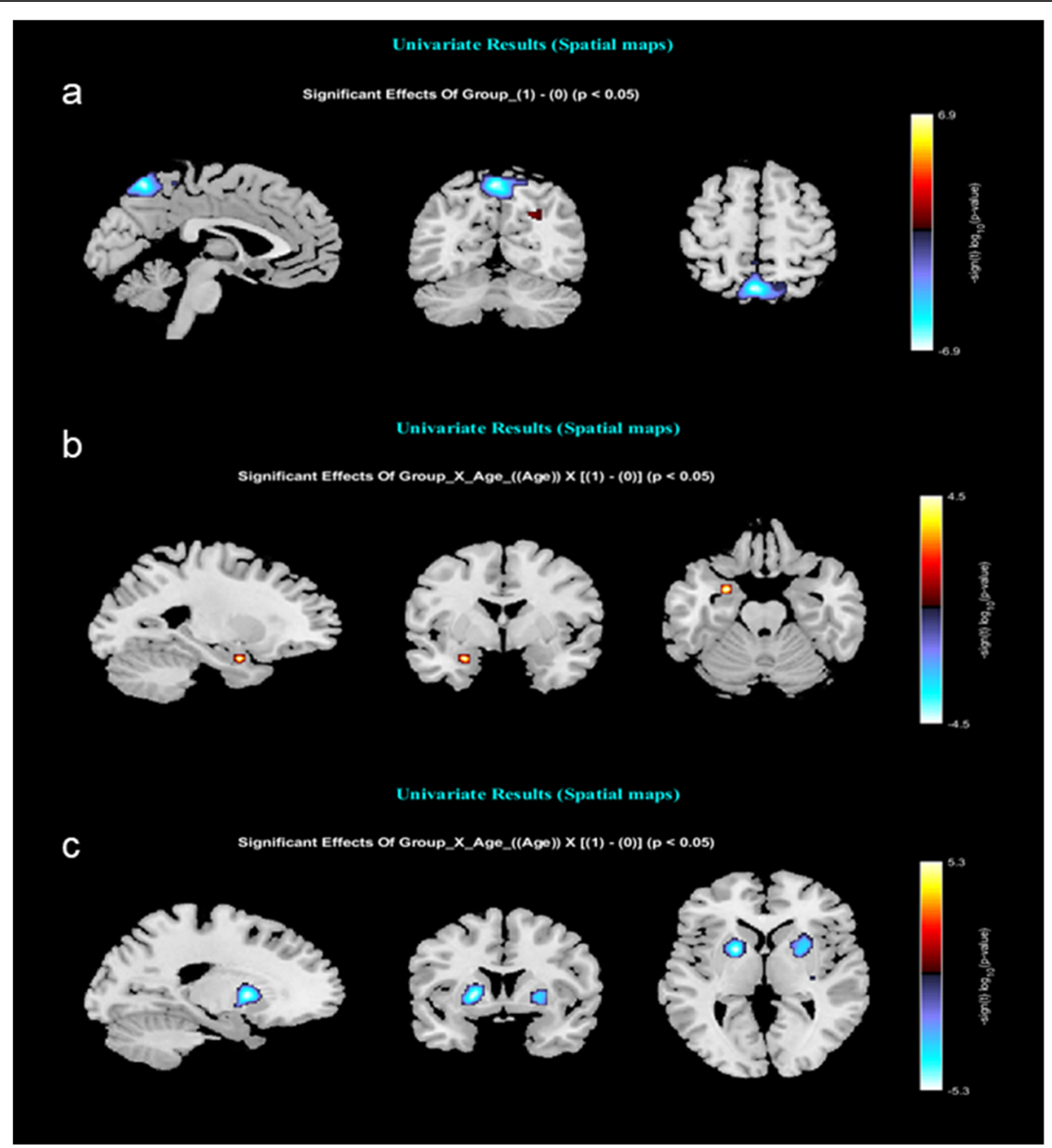

Fig. 2 Univariate results showing a) significantly lower intrinsic connectivity in the AN group in component C4, and a significant group $x$ age interaction effect in component C6 (b) and C24 (c)

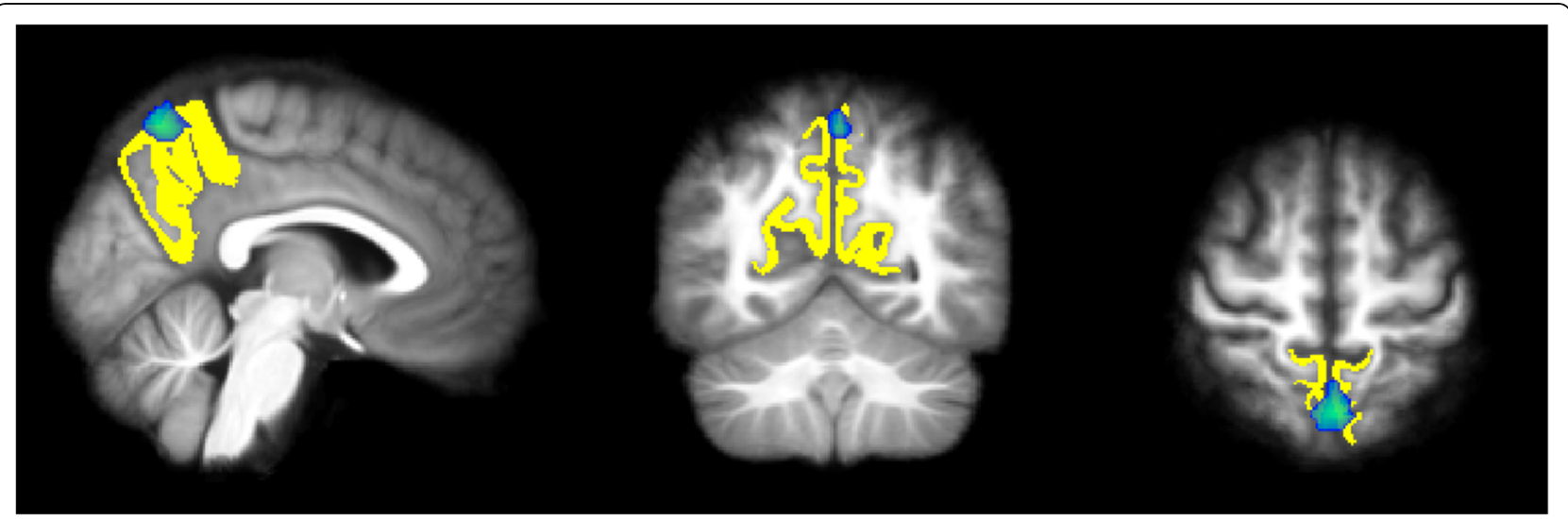

Fig. 3 The overlap between the cortical areas constituting the precuneus (yellow color) from FreeSurfer and the activation found in the restingstate fMRI analysis (blue color) 


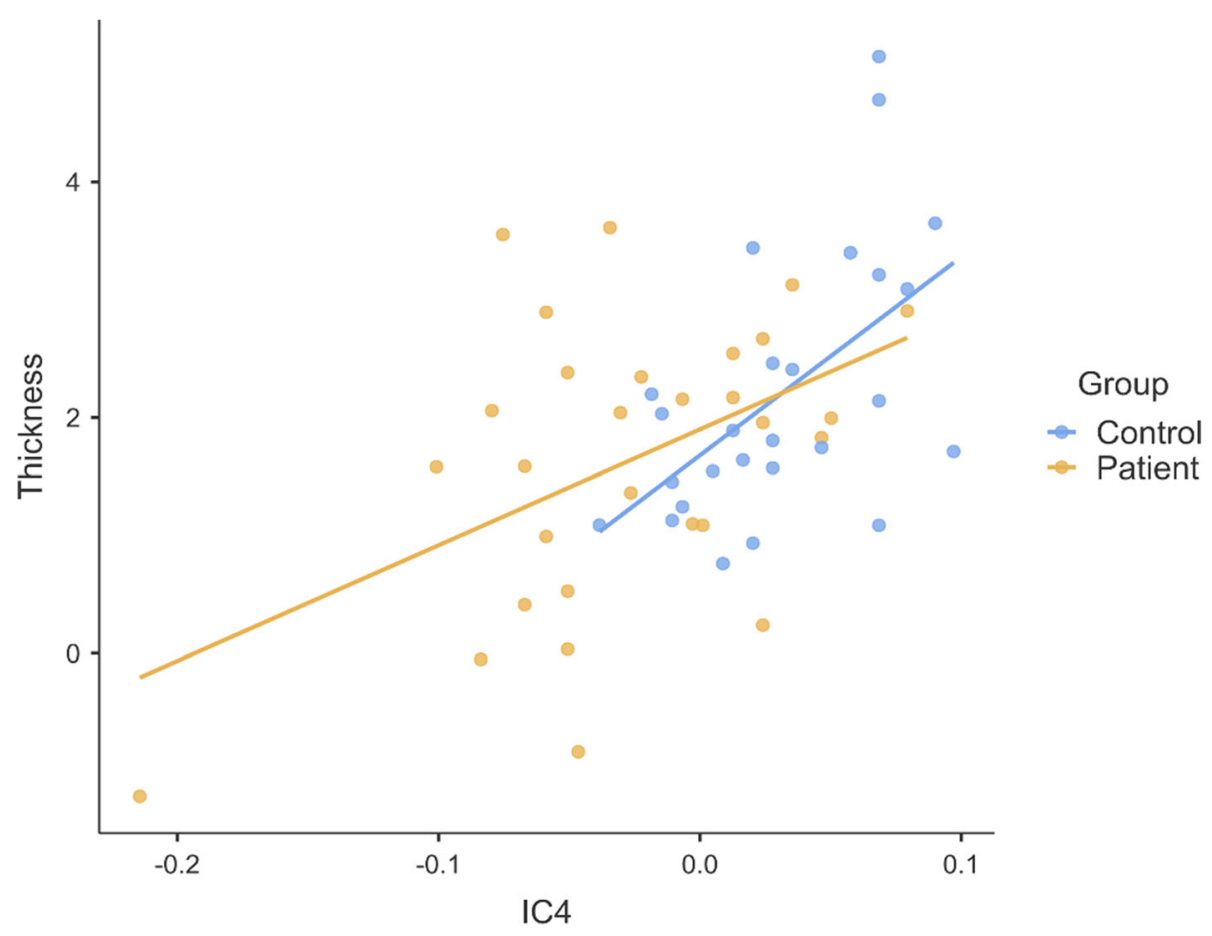

Fig. 4 Pearson correlation between mean precuneus thickness (mean of left + right precuneus) in the precuneus and the peak activation in C4. $r=.53, p<.001$ Bonferroni adjusted

regions Results from the present study did not show a significant association with the self-reported measure of "concerns about figure", as one might expect in light of previous findings.

Correlational analyses showed that connectivity in the precuneus network was associated with precuneus thickness, suggesting a cerebral structure-function link. Several studies have reported decreased volume or cortical thickness in the precuneus in AN patients [37-42], and a recent study in adult AN patients showed a relationship between precuneus thickness, reduced in their AN sample, and functional connectivity in the DMN and a central executive network [16]. A structure-function link is also found in a somatosensory network [14]. Findings from two recent studies with adolescent samples contradict this link however; Lotter et al. (2021) report global connectivity alterations that are unrelated to global GM volume [51] and Seidel et al. (2019) report a weakened relationship between measures of local characteristics of the BOLD signal and cortical thickness and volume [17]. This discrepancy may be due to the different approaches to investigating functional connectivity. As GM reduction and functional connectivity alterations is not observed in all brain regions, and may not overlap in several anatomical areas, investigating whole brain measures may mask regional relationships. Regional structure-function links may exist, and future studies should aim to investigate areas of decreased cortical volume or thickness and functional connectivity in corresponding anatomical areas.

Results from the present study show that AN patients have decreasing intra-connectivity in a hippocampus network and increasing intra-connectivity in an amygdala network with increasing age compared to HC. These results may suggest that AN disrupts normal age-related development of network intraconnectivity, expected to increase during adolescence [13]. Two studies using graph theoretical metrics to detect functional networks also found decreased connectivity in adolescent AN patients in networks resembling the two subcortical networks found in this study $[43,44]$. One of these studies tested the association with age, with no significant findings, however neither investigated the interaction effect of age and group as done in the present study. Future RSN studies should investigate the effect of age in adolescent AN patients, preferably with longitudinal sampling. Development of functional networks have been linked to pubertal status [45]. Delayed or disrupted pubertal onset is commonly found in $\mathrm{AN}$, and a possible delay in network development may be due to this. A recent review of fMRI-studies in adolescent AN suggest that 
puberty delay can affect brain maturation and lead to impaired cognitive flexibility that in turn maintains the disorder and makes it difficult to combat [46]. Pubertal status was not recorded in this study and future research should include such measures to investigate if delayed or disrupted puberty affects brain maturations in $\mathrm{AN}$.

In a previous study including the same sample [20], we found that the hippocampus may be more vulnerable to AN in terms of volume decrease compared to brain as a whole. However, correlational analyses of hippocampus volume and the hippocampus network were not significant, indicating that the structural alterations in this region were not associated with the functional alterations in RSNs. Analyses with eating disorder symptoms as covariates did not produce significant results and could thus not shed light on the mechanisms behind the interrupted development of these networks. Variables not included in this study such as hormonal levels and a broader mapping of eating disorder and comorbid symptoms could possibly explain these findings and future studies should include such measures.

Previous RSN studies of adolescent AN patients have found altered connectivity involving visuospatial networks [10], fronto-parietal networks and DMN's [11, 47]. In the present study, we did not find altered functional connectivity in such networks. The previous studies investigated a few selected networks and discrepant findings may be due to the multi-network approach in this study. Another possible explanation for the different findings in the present study may be that patients had higher BMI compared to the samples in previous studies. It is possible that functional changes in the brain vary across the different stages of AN as structural alterations do [48].

\section{Strengths and limitations}

There was no a-priori selection of cerebral regions to examine and only two RSNs were excluded from analyses, leaving analyses largely data-driven. By contrast, previous studies have mostly investigated a few selected components, perhaps discarding several relevant networks. On the other hand, it could be argued that the auditory networks excluded in the present study could have an effect on the analyses given the findings in adult patients in Scaife et al. [15] even if auditory dysfunction is not a core symptom in anorexia. Furthermore, we did not assess the effect of the varying durations of treatment preceding the resting-state scan which possibly could have an impact on cerebral network functioning.

Generally, it is difficult to disentangle the effects of starvation on cerebral functioning from the effects of acute AN because the physiological and psychological responses are overlapping [49]. The present study was not designed to answer whether the cerebral changes observed was due to AN or starvation only, and the results should be interpreted according to this. The study sample was larger compared to previous studies in the field, and with a narrow age range. The analyses were conducted with up-to-date software and methods, and we controlled for potential confounding variables like scan site and drug use and multiple comparisons. Patients were not likely to be in a catabolic phase of their illness when included in the study. All patients included were on meal plans and their BMI had been increasing since admission, reducing the confounding effects of extreme malnourishment.

In a previous review, it has been recommended to control for the effects of pubertal stage, oral contraceptives and duration of illness [50]. These types of data were not available in the present study. The use of two different MRI-scanners may confound results as the magnetic fields differ between scanners. Although site effect for AN-participants was not investigated, the nonsignificant differences across sites among $\mathrm{HC}$ participants indicate that scan site did not affect main findings in this study.

\section{Conclusion}

This study provides novel findings of age and structure related alterations in functional networks in adolescent $\mathrm{AN}$. Investigating multiple $\mathrm{RSNs}$ in a multivariate analysis increases the likelihood of detecting the most affected functional networks in $\mathrm{AN}$, indicated by results from this study to be a DMN (precuneus) network and two subcortical networks (hippocampus and amygdala). These RSNs have been implicated in previous studies in AN but have not previously been linked to structural alterations (precuneus) or age (hippocampus and amygdala). Results from this study indicate that reduced cortical thickness is associated with reduced functional connectivity in the precuneus in our adolescent sample. Furthermore results may indicate that AN disrupts normal development of RSNs involving the hippocampus and amygdala. A disruption of functional network development may contribute to the maintenance of $\mathrm{AN}$, often having a prolonged course of illness and is difficult to treat. Results from this study highlights the importance of investigating multiple networks in relationship with age, brain structure and endocrinological measures in adolescent AN patients whose functional networks are still evolving. 


\section{Supplementary Information}

The online version contains supplementary material available at https://doi. org/10.1186/s12888-021-03497-4.

Additional file 1: Suppl Fig. 1. Spatial maps of the 29 investigated components. Suppl. Fig. 2. Excluded components.

\section{Acknowledgements}

We wish to thank our colleagues at the inpatient clinics, RSS and RASP, for facilitating data collection for this study.

\section{Authors' contributions}

All authors contributed to the development of the study design. $\varnothing \mathrm{R}, \mathrm{KS}, \mathrm{TE}$ TV, PA and ADM contributed to data collection. PA conducted analyzes and ADM and TV made substantial contributions in this process. ADM drafted the manuscript in close collaboration with PA and TV. All authors gave valuable comments and suggestions to the first manuscript drafts and all authors read and approved the final version.

\section{Funding}

This project was funded by the Research Council of Norway, P.O. Box 564 NO-1327 Lysaker, Norway, program "Kvinnehelse", project number: 229142 and Helse-Nord RHF, Postboks 1445, 8038 Bodø, Project number PFP114013.

\section{Availability of data and materials}

The data that support the findings of this study are available from the corresponding author, ADM, upon reasonable request.

\section{Declarations}

\section{Ethics approval and consent to participate}

The study was approved by the Norwegian Committee for Medical and Health Research Ethics (REC), North region (protocol number 302969). Informed consent was obtained from participants and parents in the case of participants below 16 years of age. All methods used in the present study were performed in accordance with the Declaration of Helsinki.

\section{Consent for publication}

Not applicable.

\section{Competing interests}

The authors have no conflict to declare.

\section{Author details}

${ }^{1}$ Department of Psychology, Faculty of Health Sciences, UiT The Artic University of Norway, Huginbakken 32, N-9037 Troms $\varnothing$, Norway. ${ }^{2}$ Department of Clinical Medicine, Faculty of Health Sciences, UiT The Arctic University of Norway, Tromsø, Norway. ${ }^{3}$ PET Center, University Hospital of North Norway, Tromsø, Norway. ${ }^{4}$ Spaulding Rehabilitation Hospital, Boston, USA. ${ }^{5}$ Regional Department for Eating Disorders, Division of Mental Health and Addiction, Oslo University Hospital, Oslo, Norway. ${ }^{6}$ Institute of clinical Medicine, Medical Faculty, University of Oslo, Oslo, Norway. ${ }^{7}$ Department of psychology, Faculty of Social Sciences, University of Oslo, Oslo, Norway. ${ }^{8}$ Helgelandssykehuset, Mosjøen, Norway. ${ }^{9}$ Regional Center for Eating Disorders, University Hospital of North Norway, Tromsø, Norway.

Received: 12 April 2021 Accepted: 22 September 2021

\section{Published online: 06 October 202}

\section{References}

1. King JA, Geisler D, Ritschel F, Boehm I, Seidel M, Roschinski B, et al. Global cortical thinning in acute anorexia nervosa normalizes following long-term weight restoration. Biol Psychiatry. 2015;77(7):624-32 [cited 2016 Oct 7]. Available from: http://www.sciencedirect.com/science/article/pii/ S0006322314007045

2. Seitz J, Herpertz-Dahlmann B, Konrad K. Brain morphological changes in adolescent and adult patients with anorexia nervosa. J Neural Transm. 2016; 123(8):949-59 Springer Vienna; Available from: https://link.springer.com/a rticle/10.1007/s00702-016-1567-9.
3. Myrvang AD, Vangberg TR, Stedal $K, R \varnothing \varnothing$, Endestad T, Rosenvinge $J H$, et al. Cerebral cortical thickness and surface area in adolescent anorexia nervosa: Separate and joint analyses with a permutation-based nonparametric method. Int J Eat Disord. 2020; John Wiley \& Sons, Ltd; [cited 2021 Jan 5]; Advance on. Available from: https://onlinelibrary.wiley.com/doi/10.1002/ea t.23448.

4. Gaudio S, Quattrocchi CC. Neural basis of a multidimensional model of body image distortion in anorexia nervosa. Neurosci Biobehav Rev. 2012 36(8):1839-47 [cited 2016 Feb 23] Available from: http://www.sciencedirect. com/science/article/pii/S0149763412000759.

5. Chao AM, Roy A, Franks AT, Joseph PV. A Systematic Review of Taste Differences Among People With Eating Disorders. Biol Res Nurs. 22(1):82-91 SAGE Publications Inc: 2020 Jan 1 [cited 2021 Jun 17]. Available from: https://pubmed.ncbi.nlm.nih.gov/31833410/

6. Faro SH, Mohamed FB. BOLD fMRI a guide to functional imaging for neuroscientists. London: Springer; 2010. Available from: http://link.springer. com/10.1007/978-1-4419-1329-6

7. Damoiseaux JS, Rombouts SARB, Barkhof F, Scheltens P, Stam CJ, Smith SM, et al. Consistent resting-state networks across healthy subjects. Proc Nat Acad Sci U S A. 2006;103(37):13848-53. Available from: https://pubmed.ncbi. nlm.nih.gov/16945915/. https://doi.org/10.1073/pnas.0601417103.

8. Raichle ME. The Brain's Default Mode Network. Annu Rev Neurosci. 2015; 38(1):433-47 Annual Reviews Inc.;. Available from: https://www.annua Ireviews.org/doi/pdf/10.1146/annurev-neuro-071013-014030.

9. Buckner RL, Andrews-Hanna JR, Schacter DL. The brain's default network: anatomy, function, and relevance to disease. Ann N Y Acad Sci [Internet]. 2008;1124(1):1-38. Available from: https://psycnet.apa.org/record/200805643-001. https://doi.org/10.1196/annals.1440.011.

10. Favaro A, Santonastaso P, Manara R, Bosello R, Bommarito G, Tenconi E, et al. Disruption of visuospatial and somatosensory functional connectivity in anorexia nervosa. Biol Psychiatry. 2012;72(10):864-70 [cited 2015 Jul 30] Available from: http://www.sciencedirect.com/science/article/pii/ S0006322312004088

11. Boehm I, Geisler D, King JA, Ritschel F, Seidel M, Deza Araujo Y, et al. Increased resting state functional connectivity in the fronto-parietal and default mode network in anorexia nervosa. Front Behav Neurosci. 2014:8. 346 [cited 2015 Dec 1]. Available from: http://www.pubmedcentral.nih.gov/a rticlerender.fcgi?artid $=4183185 \&$ tool $=$ pmcentrez\&rendertype $=$ abstract.

12. Plana MT, Torres T, Rodríguez N, Boloc D, Gassó P, Moreno E, et al. Genetic variability in the serotoninergic system and age of onset in anorexia nervosa and obsessive-compulsive disorder. Psychiatry res [internet]. Elsevier Ireland Ltd. 2019;271:554-8 Available from: https://www.sciencedirect.com/ science/article/pii/S0165178118314021?casa token=rw6WqxCYur8AAAAA:1 H5hLB3ILgbG51P4RiOXagzrSPMRQwYNrtkHcUgoZ9oFAMdO5T5ao71990WjF8A6301_RN4bmH4

13. Stevens MC, Pearlson GD, Calhoun VD. Changes in the interaction of resting-state neural networks from adolescence to adulthood. Hum Brain Mapp. 2009;30(8):2356-66 Available from: https://onlinelibrary.wiley.com/ doi/epdf/10.1002/hbm.20673.

14. Bär K-J, de la Cruz F, Berger S, Schultz CC, Wagner G. Structural and functional differences in the cingulate cortex relate to disease severity in anorexia nervosa. J Psychiatry Neurosci. 2015;40(4):269-79. Available from: http://www.pubmedcentral.nih.gov/articlerender.fcgi?artid=4478060\&tool= pmcentrez\&rendertype=abstract. https://doi.org/10.1503/jpn.140193.

15. Scaife JC, Godier LR, Filippini N, Harmer CJ, Park RJ. Reduced Resting-State Functional Connectivity in Current and Recovered Restrictive Anorexia Nervosa. Front Psychiatry. 2017;8:30 Frontiers Media SA; [cited 2017 Nov 2]. Available from: http://www.ncbi.nlm.nih.gov/pubmed/28400737.

16. de la Cruz F, Schumann A, Suttkus S, Helbing N, Zopf R, Bär KJ. Cortical thinning and associated connectivity changes in patients with anorexia nervosa. Transl Psychiatry [Internet]. Springer Nature. 2021;11(1):95. Available from: https://doi.org/10.1038/s41398-021-01237-6. [cited 2021 Jun 11].

17. Seidel M, Borchardt V, Geisler D, King JA, Boehm I, Pauligk S, et al. Abnormal spontaneous regional brain activity in young patients with anorexia nervosa. J Am Acad Child Adolesc Psychiatry. 2019;58(11):1104-14. https:// doi.org/10.1016/j.jaac.2019.01.011

18. Gaudio S, Piervincenzi C, Zobel BB, Montecchi FR, Riva G, Carducci F, et al. Altered resting state functional connectivity of anterior cingulate cortex in drug naïve adolescents at the earliest stages of anorexia nervosa. Sci Rep. 2015;5(1):10818. https://doi.org/10.1038/srep10818. 
19. Júlíusson PB, Roelants M, Nordal E, Furevik L, Eide GE, Moster D, et al. Growth references for 0-19 year-old Norwegian children for length/height, weight, body mass index and head circumference. Ann Hum Biol. 2013; 40(3):220-7 Available from: http://www.tandfonline.com/doi/full/10.3109/03 014460.2012 .759276

20. Myrvang AD, Vangberg TR, Stedal $K, R \varnothing \varnothing$, Endestad T, Rosenvinge $J H$, et al. Hippocampal subfields in adolescent anorexia nervosa. Psychiatry Res Neuroimaging. 2018;282:24-30 Elsevier; [cited 2018 Nov 5]. Available from: https://www.sciencedirect.com/science/article/pii/S0925492718301 550?dgcid=author.

21. ICA-AROMA [Internet]. [cited 2018 Nov 8]. Available from: https://github. com/maartenmennes/ICA-AROMA

22. Pruim RHR, Mennes M, van Rooij D, Llera A, Buitelaar JK, Beckmann CF, et al. A robust ICA-based strategy for removing motion artifacts from $\mathrm{fMRI}$ data. Neuroimage. 2015;112:267-77 Academic Press; [cited 2018 Nov 8]. Available from: https://www.sciencedirect.com/science/article/pii/S1053811915001822.

23. Group ICA toolbox (GIFT and EEGIFT) [Internet]. [cited 2018 Dec 1]. Available from: http://icatb.sourceforge.net/

24. Beckmann CF, DeLuca M, Devlin JT, Smith SM. Investigations into restingstate connectivity using independent component analysis. Philos Trans R Soc Lond B Biol Sci. 2005;360(1457):1001-13 [cited 2014 Jul 10]. Available from: http://rstb.royalsocietypublishing.org/content/360/1457/1001.short.

25. Smith SM, Fox PT, Miller KL, Glahn DC, Fox PM, Mackay CE, et al. Correspondence of the brain's functional architecture during activation and rest. Proc Natl Acad Sci U S A. 2009;106(31):13040-5 [cited 2014 Jul 10]. Available from: http://www.pubmedcentral.nih.gov/articlerender.fcgi?artid= 2722273\&tool=pmcentrez\&rendertype=abstract.

26. Allen EA, Erhardt EB, Damaraju E, Gruner W, Segall JM, Silva RF, et al. A baseline for the multivariate comparison of resting-state networks. Front Syst Neurosci. 2011;5:2 [cited 2015 Jun 25]. Available from: http://www. pubmedcentral.nih.gov/articlerender.fcgi?artid=3051178\&tool= pmcentrez\&rendertype $=$ abstract.

27. Correa N, Adali T, Calhoun VD. Performance of blind source separation algorithms for FMRI analysis using a group ICA method. Neuropsychiatry (London). 2007;25(5):684-94. Available from: https://www.sciencedirect.com/ science/article/pii/S0730725X06003080?casa_token=wUzlhwAt-54AAAAA: znX587M4ng2mrng_YeHerRd9_TsCr_ZN_bYHCf7DmzOCLIPZJxEkteRPYbG6La GXnX1NtmVaMypB. https://doi.org/10.1016/j.mri.2006.10.017.

28. Fairburn CG, Beglin S. Eating disorder examination questionnaire (EDE-Q 6. 0). In: Fairburn CG, editor. Cognitive Behavior Therapy and Eating Disorders. New York: Guilford Press; 2008. p. 309-13.

29. FreeSurfer [Internet]. Available from: https://surfer.nmr.mgh.harvard.edu/

30. Fischl B, Salat DH, Busa E, Albert M, Dieterich M, Haselgrove C, et al. Whole brain segmentation: automated labeling of neuroanatomical structures in the human brain. Neuron. 2002;33(3):341-55. Available from: https://www. sciencedirect.com/science/article/pii/S089662730200569X. https://doi.org/1 0.1016/S0896-6273(02)00569-X

31. Fischl B, Van Der Kouwe A, Destrieux C, Halgren E, Ségonne F, Salat DH, et al. Automatically parcellating the human cerebral cortex. Cereb Cortex. 2004;14(1):11-22. https://doi.org/10.1093/cercor/bhg087.

32. Desikan RS, Ségonne F, Fischl B, Quinn BT, Dickerson BC, Blacker D, et al. An automated labeling system for subdividing the human cerebral cortex on MRI scans into gyral based regions of interest. Neuroimage. 2006;31(3):96880 Academic Press; [cited 2020 Jun 12]Available from: https://www. sciencedirect.com/science/article/pii/S1053811906000437?casa_token= 7RPxkhyy-gQAAAAA:NYT7BJhztlhNqRL1sE9koRkgs5_g9dvUxvGUmb4 yEdeiSO1VzgLP-W4w3K6d4rRgW_kT6scQw7-J.

33. Utevsky AV, Smith DV, Huettel SA. Precuneus is a functional core of the default-mode network. J Neurosci. 2014;34(3):932-40 [cited 2021 Mar 30]; Society for Neuroscience: Available from: http://www.fmrib.ox.ac.uk/fsl/.

34. Mohr HM, Zimmermann J, Rö Der C, Lenz C, Overbeck G, Grabhorn R. Separating two components of body image in anorexia nervosa using fMRI. Psychol Med. 2009;40:1519-29 Available from: https://scholar.google.com/ scholar_url?url=https://search.proquest.com/openview/aa782871e3ba2e459231 8ea0ec930e00/1\%3Fpq-origsite\%3Dgscholar\%26cbl\%3D35753\%26casa_ token\%3DicgxeQvWynEAAAAA:PD4ocde0IZucKSxJaPT1DCBgsU5yAHCzHRS3 vPfwgEhYB_bGPOOVofdVhwjg6uR5Fyye6CwyYqzP\&hl=no\&sa=T\&oi=gsbggp\&ct=res\&cd=0\&d=18188110132047744272\&ei=HVbMYNSOGIXSmA GuvJ7YDw\&scisig=AAGBfm0ulKIUhcSvlg5nd_SWNrPKh2ot5Q.

35. McFadden KL, Tregellas JR, Shott ME, GKW F. Reduced salience and default mode network activity in women with anorexia nervosa. J Psychiatry
Neurosci. 2014;39(3):178-88 [cited 2015 Jul 30]. Available from: http://www. pubmedcentral.nih.gov/articlerender.fcgi?artid=3997603\&tool= pmcentrez\&rendertype=abstract.

36. Lee S, Ran Kim K, Ku J, Lee JH, Namkoong K, Jung YC. Resting-state synchrony between anterior cingulate cortex and precuneus relates to body shape concern in anorexia nervosa and bulimia nervosa. Psychiatry Res Neuroimaging. 2014;221(1):43-8 Elsevier; [cited 2021 Jun 17]. Available from: https://www.sciencedirect.com/science/article/pii/S0925492713003120.

37. Nickel K, Joos A, Tebartz van Elst L, Matthis J, Holovics L, Endres D, et al. Recovery of cortical volume and thickness after remission from acute anorexia nervosa. Int J Eat Disord. 2018;51(9):1056-69 [cited 2019 Aug 8]. John Wiley \& Sons, Ltd; Available from: http://doi.wiley.com/10.1002/eat.22918.

38. Leppanen J, Sedgewick F, Cardi V, Treasure J, Tchanturia K. Cortical morphometry in anorexia nervosa: An out-of-sample replication study. Eur Eat Disord Rev. 2019;erv:2686 John Wiley \& Sons, Ltd [cited 2019 Aug 15]. Available from: https://onlinelibrary.wiley.com/doi/abs/10.1002/erv.2686.

39. Bomba M, Riva A, Morzenti S, Grimaldi M, Neri F, Nacinovich R. Global and regional brain volumes normalization in weight-recovered adolescents with anorexia nervosa: preliminary findings of a longitudinal voxel-based morphometry study. Neuropsychiatr Dis Treat. 2015;11:637-45 Dove Press [cited 2016 Apr 6]. Available from: https://www.dovepress.com/global-andregional-brain-volumes-normalization-in-weight-recovered-ad-peerreviewed-article-NDT.

40. Gaudio S, Nocchi F, Franchin T, Genovese E, Cannatà V, Longo D, et al. Gray matter decrease distribution in the early stages of Anorexia Nervosa restrictive type in adolescents. Psychiatry Res Neuroimaging. 2011;191(1):2430 [cited 2016 Feb 23]. Available from: http://www.sciencedirect.com/ science/article/pii/S0925492710002258.

41. Frank GKW. Neuroimaging and eating disorders. Curr Opin Psychiatry. 2019; 32(6):478-83. Available from: https://journals.lww.com/co-psychiatry/ FullText/2019/11000/Neuroimaging_and_eating_disorders.3.aspx?casa_ token $=\mathrm{VEX} 2 \mathrm{ObaEiLUAAAAA} 9 \mathrm{FJ}$ DqtVxtYIB9WsnCfhXpYKQYgvFhjUtYjIm9SFInS550bVdgfl5s3TS7zUBDMwf62 h8d3wdfTToSoTMyb8mBdDUDb5PARoQ. https://doi.org/10.1097/YCO. 0000000000000544

42. Yue L, Wang Y, Kaye WH, Kang Q, Bin HJ, EFC C, et al. Structural alterations in the caudate nucleus and precuneus in un-medicated anorexia nervosa patients. Psychiatry Res - Neuroimaging. 2018;281:12-8 Available from: https://www.sciencedirect.com/science/article/pii/S0925492718300969?casa_ token=rUw-FULXoPMAAAAA:opFoX40NBOa9EMOBvrSvPORYqPNCs7kglwl0Jy57UmcTT_jjSCsyF2_-L3QyfjvtVokAM3elxw4.

43. Ehrlich S, Lord AR, Geisler D, Borchardt V, Boehm I, Seidel M, et al. Reduced functional connectivity in the thalamo-insular subnetwork in patients with acute anorexia nervosa. Hum Brain Mapp. 2015;36(5):1772-81 Available from: http://doi.wiley.com/10.1002/hbm.22736.

44. Geisler D, Borchardt V, Lord AR, Boehm I, Ritschel F, Zwipp J, et al. Abnormal functional global and local brain connectivity in female patients with anorexia nervosa. J Psychiatry Neurosci. 2016;41(1):6-15 Canadian Medical Association; [cited 2018 may 29]. Available from: http://www.ncbi. nlm.nih.gov/pubmed/26252451.

45. Gracia-Tabuenca Z, Moreno MB, Barrios FA, Alcauter S. Development of the brain functional connectome follows puberty-dependent nonlinear trajectories. Neuroimage. 2021;229:117769 Academic Press Inc.; [cited 2021 Apr 5]. Available from: https:/www.sciencedirect.com/science/article/pii/S105381192100046X.

46. Olivo G, Gaudio S, Schiöth HB. Brain and Cognitive Development in Adolescents with Anorexia Nervosa: A Systematic Review of fMRI Studies. Nutrients. 2019;11(8):1907 MDPI AG; [cited 2020 Aug 14]. Available from: https://www.mdpi.com/2072-6643/11/8/1907.

47. Boehm I, Geisler D, Tam F, King JA, Ritschel F, Seidel M, et al. Partially restored resting-state functional connectivity in women recovered from anorexia nervosa. J Psychiatry Neurosci. 2016;41(6) Available from: https:// www.ncbi.nlm.nih.gov/pmc/articles/PMC5082508/.

48. Bernardoni F, King JA, Geisler D, Stein E, Jaite C, Nätsch D, et al. Weight restoration therapy rapidly reverses cortical thinning in anorexia nervosa: $A$ longitudinal study. Neuroimage. 2016;130:214-22 Academic Press; [cited 2018 Apr 9]. Available from: https://www.sciencedirect.com/science/article/ pii/S1053811916001014.

49. Zipfel S, Giel KE, Bulik CM, Hay P, Schmidt U. Anorexia nervosa: aetiology, assessment, and treatment. Lancet Psychiatry. 2015;2(12):1099-111. Elsevier Ltd; Available from: https://www.sciencedirect.com/science/article/abs/pii/ S2215036615003569. https://doi.org/10.1016/\$2215-0366(15)00356-9. 
50. King JA, Frank GKW, Thompson PM, Ehrlich S. Structural Neuroimaging of Anorexia Nervosa: Future Directions in the Quest for Mechanisms

Underlying Dynamic Alterations. Biol Psychiatry. 2018;83(3):224-34 Elsevier; [cited 2019 Mar 18] Available from: https://www.sciencedirect.com/ science/article/pii/S000632231731898X?via\%3Dihub.

51. Lotter LD, von Polier G, Offermann J, Buettgen K, Stanetzky L, Eickhoff SB, et al. Recovery-associated resting-state activity and connectivity alterations in Anorexia nervosa. Biol Psychiatry Cogn Neurosci Neuroimaging [Internet]. Elsevier Inc. 2021;(22). Available from: https://doi.org/10.1016/j.bpsc.2021.03. 006.

\section{Publisher's Note}

Springer Nature remains neutral with regard to jurisdictional claims in published maps and institutional affiliations.

Ready to submit your research? Choose BMC and benefit from:

- fast, convenient online submission

- thorough peer review by experienced researchers in your field

- rapid publication on acceptance

- support for research data, including large and complex data types

- gold Open Access which fosters wider collaboration and increased citations

- maximum visibility for your research: over $100 \mathrm{M}$ website views per year

At $\mathrm{BMC}$, research is always in progress.

Learn more biomedcentral.com/submissions 